\title{
X-ray Micro Tomography Study of Internal Defects of Electron Beam Melted Ti6Al4V and Their Effect on Fatigue Behavior
}

Viktor Sandell $^{\mathrm{a}}{ }^{*}$, Thomas Hansson ${ }^{\mathrm{b}}$, Fredrik Forsberg ${ }^{\mathrm{c}}$, Henrik Lycksam $^{\mathrm{c}}$, Pia Åkerfeldt ${ }^{\mathrm{a}}$, Marta-Lena Antti $^{\mathrm{a}}$

${ }^{\mathrm{a}}$ Division of Material Science, Luleå University of Technology, 97187 Luleå, Sweden

b GKN Aerospace Sweden AB, 46138 Trollhättan, Sweden

${ }^{\mathrm{c}}$ Division of Fluid and Experimental Mechanics, Luleå University of Technology

* viktor.sandell@ltu.se

\section{$\underline{\text { Abstract }}$}

In this work, the fatigue behaviour of Ti6Al4V manufactured using electron beam melting, its dependency on porosity, distance from the base plate and build layer height were investigated. XCT scans of the fatigue sample gauge lengths were correlated to SEM investigations of the fracture surfaces. A comparison between the top and bottom halves of the builds in terms of defect population and fatigue behaviour was also made. Larger pores were detected in samples with a larger build layer height and lower position in the build chamber. Results also indicate that part geometry and pore location, specifically closeness to the surface, are important factors regarding the initiation location of fatigue fractures at $1 \%$ strain. Furthermore, a fatigue critical lack of fusion defect was undetectable in the XCT scan.

\section{$\underline{\text { Introduction }}$}

Electron beam melting $(\mathrm{EBM})$ is a powder bed fusion $(\mathrm{PBF})$ additive manufacturing $(\mathrm{AM})$ technique that is currently growing in terms of the number of production units, as well as volume, produced [1].

Life-limiting fatigue behaviour of additive manufactured materials depends on four factors; microstructure, surface finish, residual stress and internal defects [2]. Lately, several attempts at investigating these factors with the aim to predict fatigue behaviour have been made [3-6]. For surface polished powder bed fusion produced components internal defects such as lack of fusion, gas porosity or inclusions are known to be the primary source for fatigue failure initiation [2]. Furthermore, it has been shown that the spatial distribution of internal defects plays an important role in fatigue performance [6]. The most common way of characterizing internal defects is the increasingly popular non-destructive imaging technique micro X-ray computed tomography (XCT) [3-5,7]. Due to the strong X-ray attenuation contrast between voids and metal, XCT is very suitable for this application [8]. Defects originate from either the feedstock material or the manufacturing process. Spherical porosity and high-density inclusions come from the powder used for building [9] whereas non-spherical pores are process related [10]. Defects reduce the overall lifetime of a component compared to a theoretical, defect-free, component since defects themselves acts as micro-cracks and initiation sites for further fatigue damage propagation [11, 12]. Fracture generally starts at defects that are connected to or very close to the surface of the material [13, 14]. If these are eliminated or reduced through machining and polishing or other surface treatments internal pores can become fracture inducing $[14,15]$.

Another factor that could be important for fatigue behaviour is the location-dependent material properties at different build heights. This difference can be due to microstructure variation or variation in the distribution of pores with build height as was demonstrated by Seifi et. al. [7]

To predict the effect of the porosity distribution on fatigue behaviour it is necessary to carry out porosity sensitive modelling. One way to do this is using fracture mechanics and knowledge about the pore size and positions to rank pores in how likely they are to cause a fatigue fracture. There are several ways to rank defects. Romano and Beretta [3,4] used the Murakami $\sqrt{ }$ area parameter [11] together with Kitagawa-Takahashi diagrams to predict fatigue strength of SLM produced AlSi10Mg and Ti6Al4V. Tammas-Williams et. al. [16] ranked defects in Ti6Al4V in four ways:

i. Size (volume or projected area).

ii. Varea parameter.

iii. Weighted stress concentration factor based on pore size, location, aspect ratio and local surrounding.

iv. Local FEM analysis of maximum plastic stress-strain concentration.

Romano [4] and Tammas-Williams [16] based their ranking on X-ray micro computed tomography (XCT) data from the test specimen. The present work uses XCT data of EBM produced Ti6Al4V fatigue samples to do a simple size ranking in an attempt to predict the site of fatigue crack initiation.

\section{Experimental Methods}

The investigated material was build in two separate builds at AIM Sweden, Sweden in an Arcam Q20plus. These are referred to as build A and B. Three vertically built square bars with sides $15 \times 15 \mathrm{~mm}$ and a building height of $180 \mathrm{~mm}$ were manufactured using a $50 \mu \mathrm{m}$ (build A) or $90 \mu \mathrm{m}$ (build B) layer thickness totalling six bars. Both builds used rotational hatching with 67,5 degree of rotation between layers. The hatching uses the same current and accelerating voltage for both builds so the deeper melt pool necessary in build B is produced by a slower scan speed and a slightly defocused beam. The speed function was set to 60 for build A and 32 for build B.

All XCT scans were carried out in a Zeiss Xradia 510 Versa X-ray micro tomograph from Carl Zeiss X-ray Microscopy, USA, using a cone beam set-up. The scan settings and resulting voxel sizes can be seen in table 1 .

\begin{tabular}{|c|c|c|c|c|c|c|}
\hline $\begin{array}{l}\text { Field of } \\
\text { view } \\
(\mathrm{mm})\end{array}$ & $\begin{array}{l}\text { Voxel size } \\
(\mu \mathrm{m})\end{array}$ & $\begin{array}{l}\text { Tube } \\
\text { Voltage } \\
(\mathrm{kV})\end{array}$ & \begin{tabular}{|l|}
$\begin{array}{l}\text { Tube } \\
\text { output } \\
(\mathrm{W})\end{array}$ \\
\end{tabular} & \begin{tabular}{|l}
$\#$ \\
Projections
\end{tabular} & \begin{tabular}{|l} 
Exposure \\
time \\
$(\mathrm{s})$
\end{tabular} & $\begin{array}{l}\text { Scan } \\
\text { time } \\
(\mathrm{h})\end{array}$ \\
\hline 17,4 & 8,5 & 140 & 10 & 1601 & 9 & 5,5 \\
\hline
\end{tabular}

The powder used for both builds was pre-alloyed, plasma atomized, Ti6A14V powder from AP\&C, Canada. The powder was from regular production cycles and thus consisted of a blend of fresh and recycled powder. Particle sizes (equivalent diameter) ranged from 2,9-46,4 $\mu \mathrm{m}$ with a few non-spherical particles likely due to the powder recycling.

From each Ti6Al4V bar two fatigue samples, high (H) and low (L), were machined making the total number of samples twelve. The machining was carried out at Metcut Research Inc., USA. The sample dimensions can be seen in figure 1. This further separates the samples from build A and B into four groups depending on if they were situated at the bottom (AL and BL) or at the top (AT and BT) of the build. Each group contains three samples. A summary of the samples and their fatigue behaviour can be found in table 2. After machining, the samples gauge lengths were scanned using XCT. 


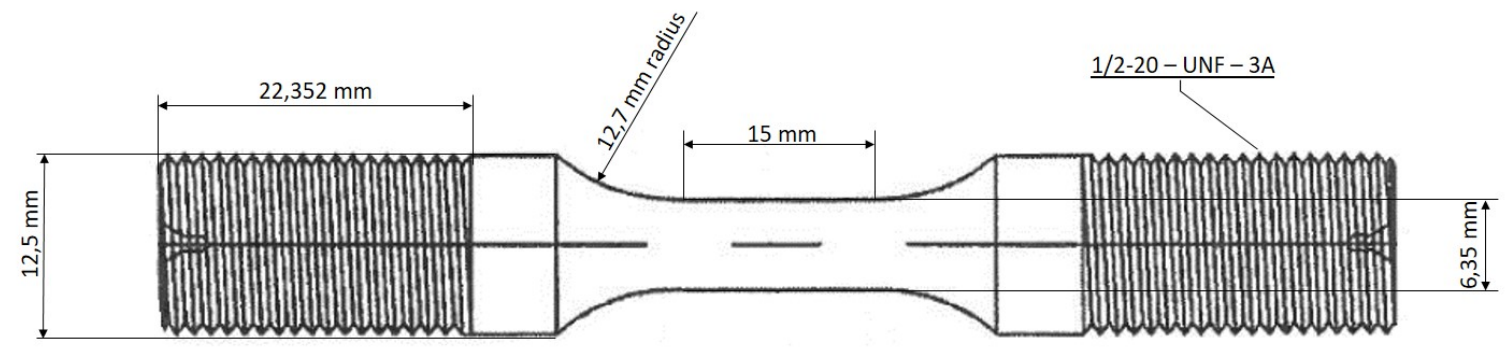

Figure 1: Sample geometry.

Three-dimensional data was reconstructed from radio-graphic projections using filtered back projection and a subsequent beam hardening correction. The data was analyzed using Dragonfly PRO, Object Research Systems, Canada. Segmentation of the closed porosity was carried out in several steps. First, the background was removed and a 3x3x3 median filter was applied to reduce noise and better include volume effects than would be possible using a two-dimensional filter. Secondly, a two-dimensional histogram is plotted combining a grey scale histogram of the material on one axis and a Sobel edge detection filtered copy of the same data on the other axis. Finally, the information is clustered using K-Means with three clusters. These three clusters typically correspond to the outer surface of the samples, the metal and the pores respectively.

\begin{tabular}{|c|c|c|c|c|c|}
\hline Sample & \begin{tabular}{||l|} 
\\
Pores
\end{tabular} & $\begin{array}{l}\text { Initiator } \\
\text { Size }(\mu m)\end{array}$ & $\begin{array}{l}\text { Initiator Percentile } \\
(\%)\end{array}$ & \begin{tabular}{|l||}
$\#$ \\
Cycles \\
\end{tabular} & $\begin{array}{l}\text { Mean \# } \\
\text { Cycles }\end{array}$ \\
\hline AH1 & 3526 & 83,6 & 97,4 & 18340 & 18676 \\
\hline $\mathrm{AH} 2$ & 2713 & 77,3 & 95,4 & 18975 & - \\
\hline AH3 & 4342 & 68,9 & 93,6 & 18712 & $\mid$ \\
\hline AL1 & 3959 & 213 & 100 & 12334 & 17497 \\
\hline AL2 & 5227 & 80,0 & 96,4 & 20763 & - \\
\hline AL3 & 4046 & 101,3 & 99,4 & 19396 & - \\
\hline BH1 & 3045 & 118,6 & 99,8 & 16935 & 17083 \\
\hline $\mathrm{BH} 2$ & 3149 & 90,5 & 95,5 & 18672 & - \\
\hline $\mathrm{BH} 3$ & 3994 & 99,8 & 98,8 & 15642 & - \\
\hline BL1 & 3838 & 108,1 & 99,0 & 15514 & 16021 \\
\hline BL2 & 4026 & 116,6 & 99,6 & 16022 & $\mid$ \\
\hline BL3 & 3831 & 79,5 & 90,63 & 16527 & $\mid$ \\
\hline
\end{tabular}

For the fatigue bars, the original voxel size was $8,5 \mu \mathrm{m}$ but due to the filtering, the effective resolution limit was $25.5 \mu \mathrm{m}$. Segmented volumes smaller than the volume of a cube with sides equal to the effective resolutions were considered as uncertain measurements and were excluded from the data. Strain-controlled fatigue testing was carried out in ambient temperature at GKN Aerospace Sweden AB, Sweden. All samples were tested at 1\% strain and with an R-ratio of -1. Fracture surface analysis was done using a JEOL JSM-IT300LV scanning electron microscope.

\section{$\underline{\text { Results \& Discussion }}$}

XCT data

Figure 2 shows defect distributions of pores segmented in the XCT scans of the gauge sections of the 12 fatigue test specimen. The size of markings is proportional to the equivalent pore diameter of the projected area along the load axis of the specimen. The ten largest pores in each sample are marked in orange and in the case that the fracture initiated from a segmented pore that pore is highlighted in red. Furthermore, there were fracture initiating pores that were not segmented by the segmentation algorithm, either because they were half pores open to the surface or because they were located outside of the gauge length. Such pores were measured using SEM fracture images and plotted in blue. Finally, there was one initiation from a Lack of Fusion (LOF) defect. This defect is marked with a green star. An analysis of the XCT data presented in figure 2 indicates that the overall distribution of pores in the plane perpendicular to the sample load axis is random and rather homogeneous. This was expected since the scanned volume was machined from a region of material in the middle of the hatch, far from any scanning inhomogeneity such as edges or contours.

As seen in histograms of the distributions (figure 3) of the defects in build A and build B, build B with thicker build layers has more large pores than build A. Looking at the exponential probability plot (Q-Q Plot) of the size distribution of the 12 samples (figure 3 ) this difference is further highlighted. Even though the difference is small below the $50^{\text {th }}$ percentile of pores the tail end of the distributions shows a gap in size between the largest pores in the two builds. The difference is the larger melt pool depth in build B compared to build A due to a difference in build speed and thus energy input density. This increases the distance a gas bubble has to rise through the melt before breaching the surface and escaping into the build chamber. One theory is that since larger bubbles experience larger buoyancy force and thus higher rise speeds in the melt than small bubbles it could allow them to faster escape more shallow melt pools prior to solidification [17]. Marangoni flow could also increase bubble retention at higher temperatures [17]. Even though the higher energy input has been suggested elsewhere to reduce the average size of gas pores [18] due to remelting of underlying layers it is likely that this effect was offset by the large difference in the total melt pool depth in combination with flow dynamics in the melt pool between builds in this study.

The left tails in figure 3 are not lined up with the right tail and the pores are thus not exponentially distributed for all segmented porosity. This is likely an effect of the limited resolution as defects are not detected or detected but too small and thus considered as uncertain measurements by the segmentation algorithm, closer to the resolution limit. Disregarding that, the points over a threshold does line up. This corresponds to observations on SLM produced AlSi10Mg studied by Romano et. al. [4] and implies that it is possible to model the distribution of the largest pores with a Gumbel (exponential base) distribution employing extreme value theory [4]. 


\section{$\mathrm{AH} 1$}
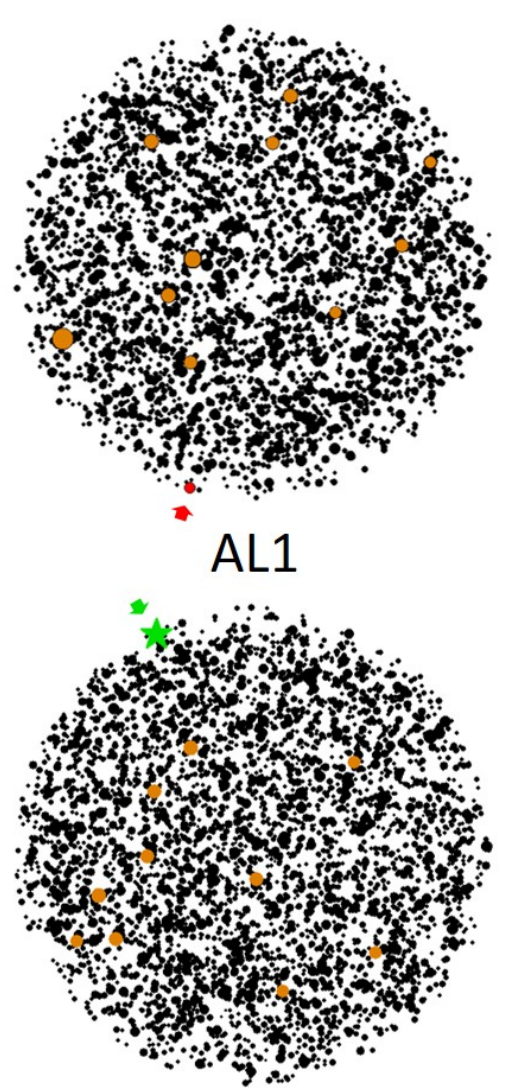

$\mathrm{BH} 1$

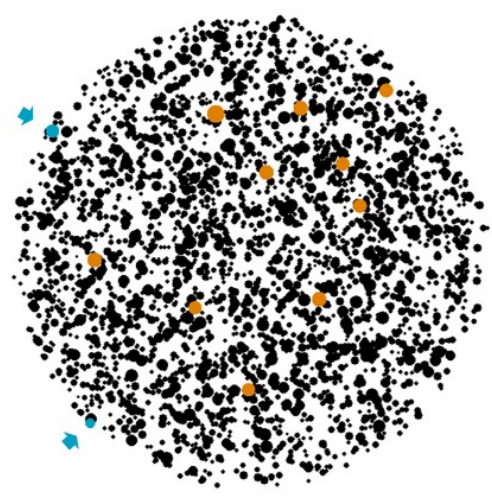

BL1

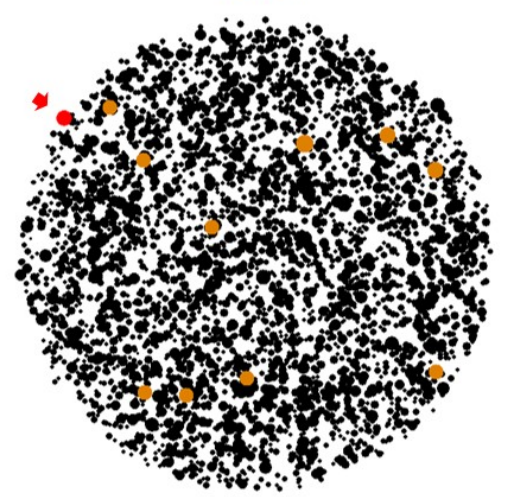

$\mathrm{AH} 2$
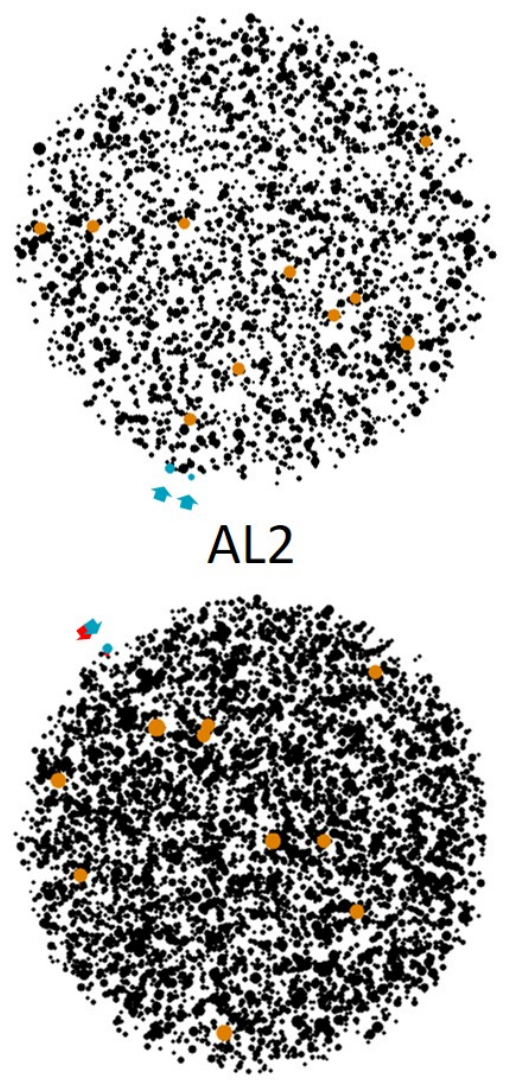

$\mathrm{BH} 2$

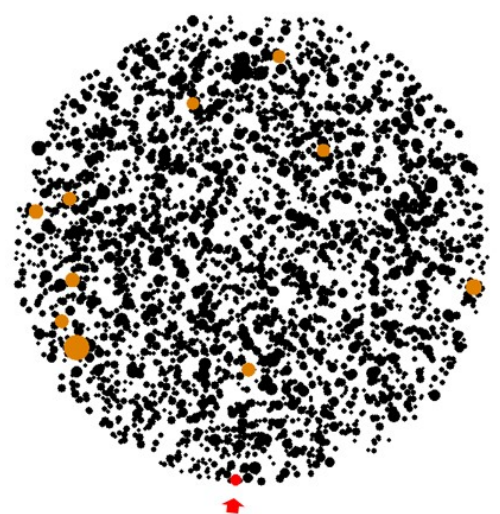

BL2

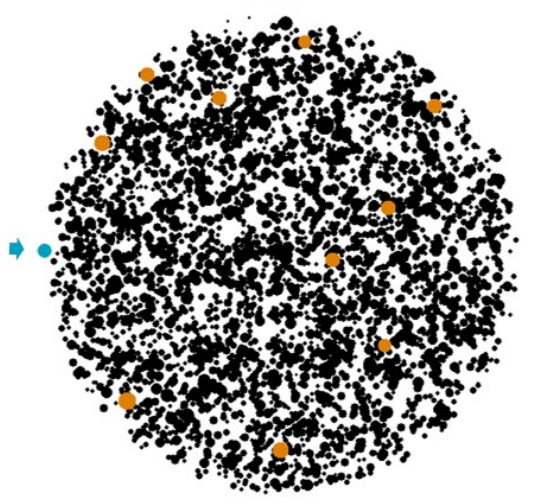

$\mathrm{AH} 3$

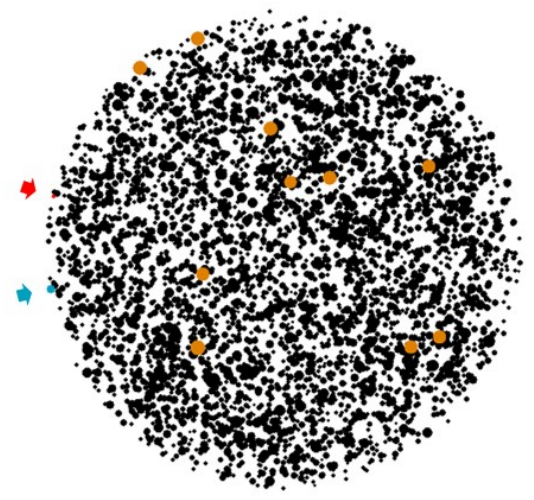

AL3

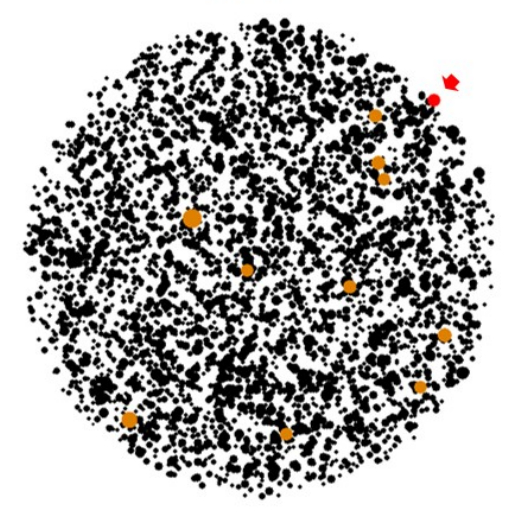

$\mathrm{BH} 3$

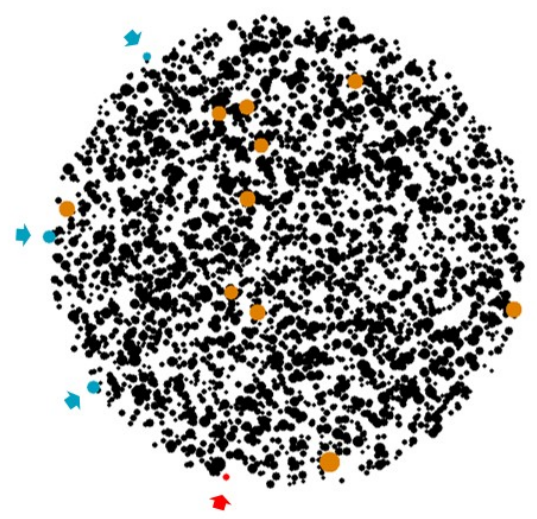

BL3

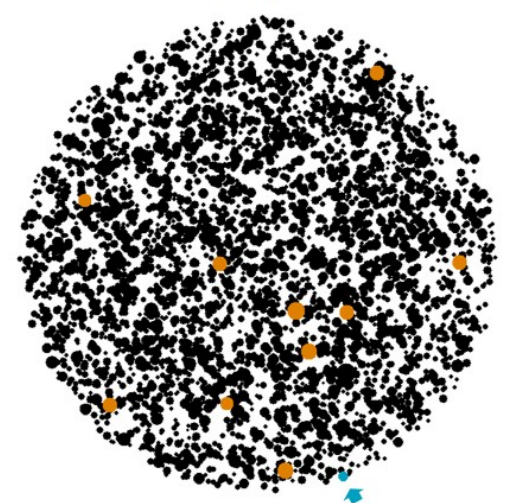



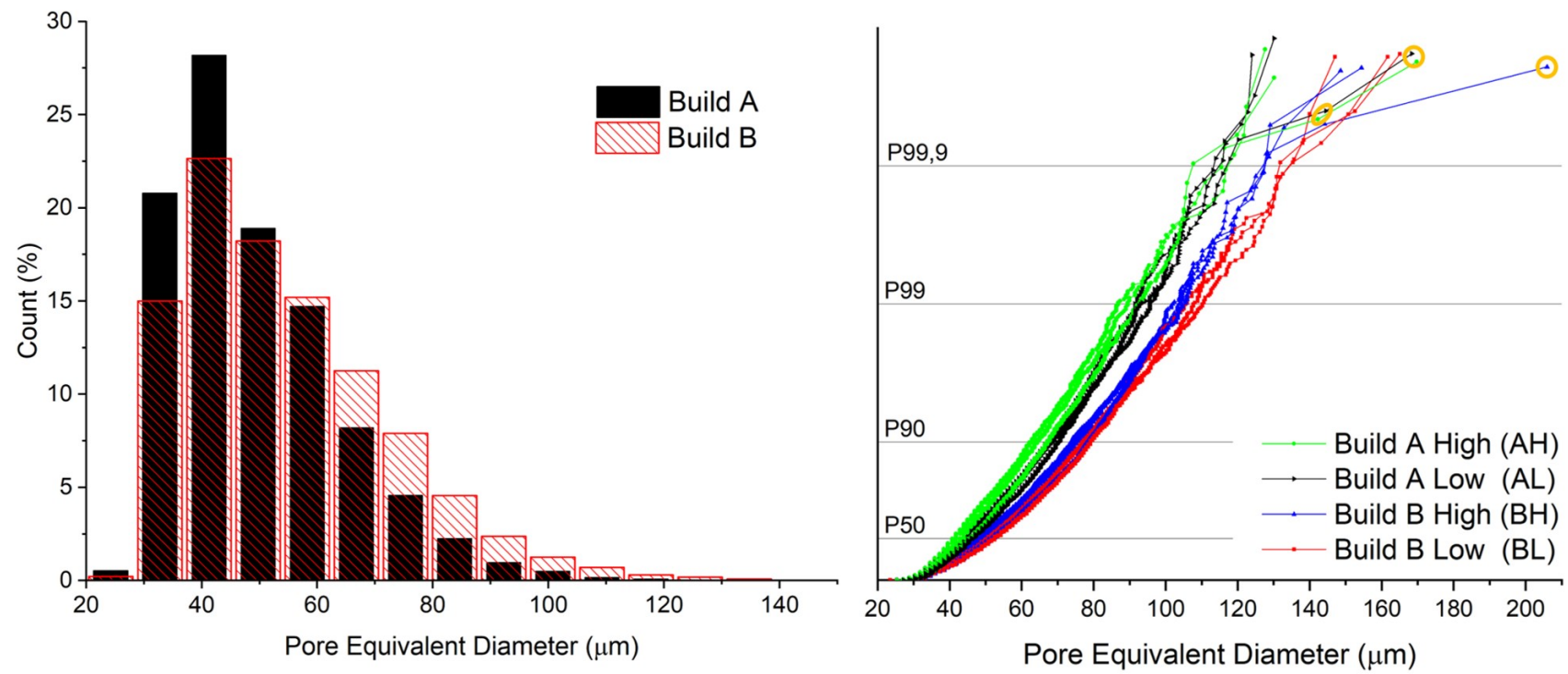

Figure 3: Left: Histogram comparing the pore size distributions in the two builds. Right: Exponential Q-Q plots of the twelve samples. Colours show build, A or B, and position in the build chamber, high or low.

There are a few large pores that are outliers in the data marked by yellow circles in figure 3 . These pores have in common that they are flattened i.e. thinner in the load direction of the sample in relation to their area perpendicular to the load axis. Since the equivalent diameter in figure 3 was calculated from the projected area of the pore along the load axis the diameter of these pores is overestimated. Using any linear units for defect size that is derived from the volume rather than the projected surface area (such as the cube root of the volume) these outlier line up with the rest of the data. It is the pore area in the plane of greatest stress (together with the closeness to the surface) that is the most important factor for determining the probability of crack initiation [11]. Thus defining defect size from the projected area appears superior to defining it from the volume since flat pores in the plane of stress could be especially dangerous in fatigue.

In order to compare the pore distribution between different heights of the build, it is possible to further separate the data in figure 3 into samples machined from the high and low parts of the builds as can be seen in figure 3. Doing this it is possible to read that there are more large pores in the low part of both builds. Even though the difference is small (about $10 \mu \mathrm{m}$ in relative diameter at the 99 th percentile of build B) or even negligible, the trend is clear. The reason for this difference is unclear and could be a subject for future studies. A difference such as this can be a problem since a larger total pore volume gives a lower fracture toughness [7] and since fracture mechanical models usually assume homogeneous fracture toughness.

Fatigue tests

The result of fatigue testing can be seen in table 2. Eleven samples fractured at pores and one at a LOF defect. All except for two fracture initiating pores (from the same sample, BL3) could be identified in the XCT data. The two that were not identifiable were positioned outside of the XCT scanned volume.

There was little spread in the data with build B showing a slightly shorter life than build A. The only outlier was sample AL1 fracturing at a LOF defect that lasted 12334 cycles. As previously stated the position of this defect is marked with a green star in figure 2 and is imaged using SEM in figure 4 where it is compared to a typical pore induced initiation. With size at least three times that of an average initiating pore it is clear why sample AL1 broke early.
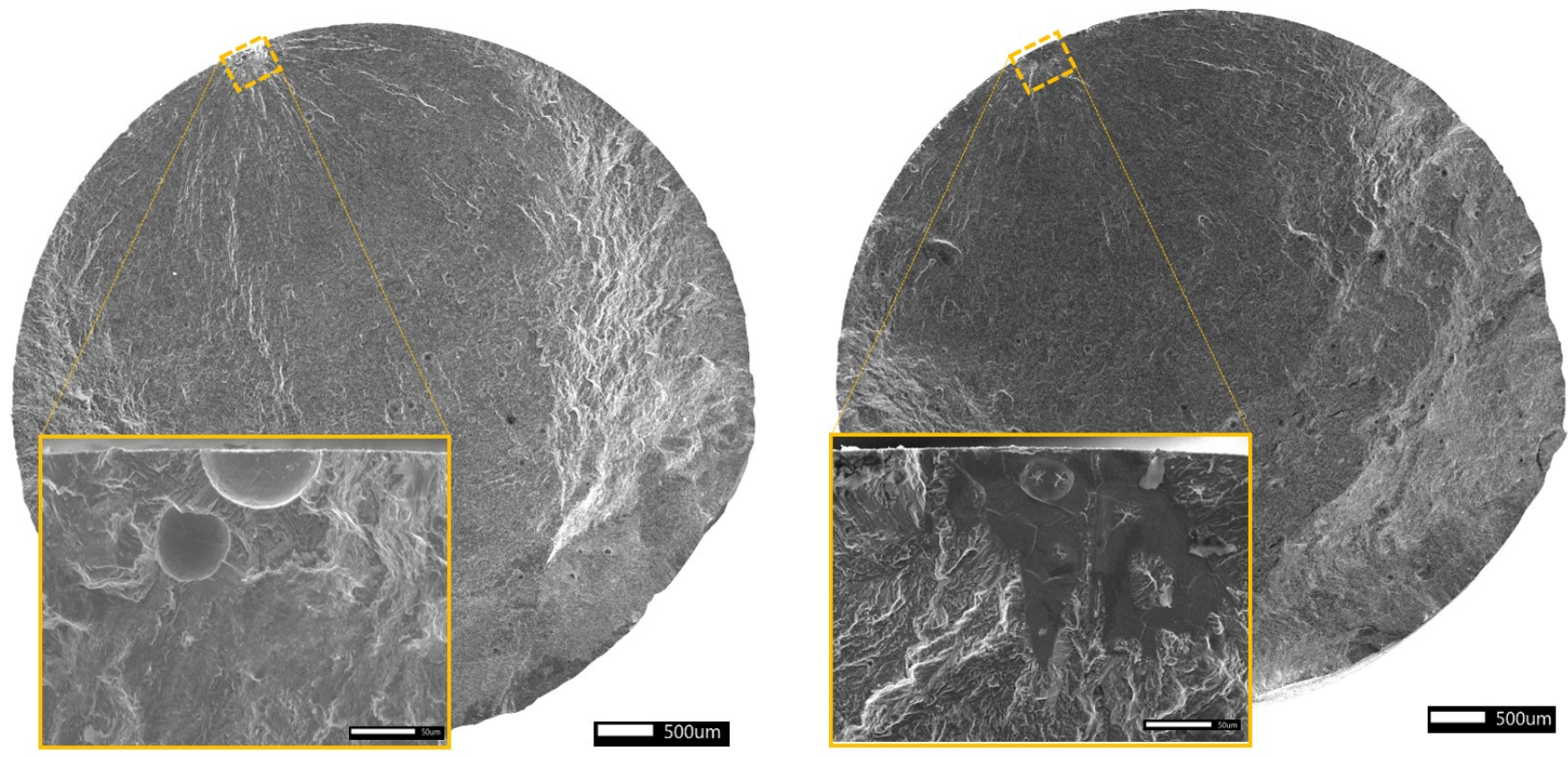

Figure 4: Left: Fracture surface of sample AL2 originating at a pair of pores. Right: Fracture surface of sample AL1 originating at a LOF defect.

The fracture initiating LOF could not be seen in the XCT data. This is likely because of its morphology being flat with a thickness below the resolution of the XCT scan. It is also worth noting that the surface open pore in figure 4 is marked blue in figure 2 and was thus not detected in the segmentation whilst the smaller closed pore was. To avoid missing such critical features during XCT analysis a more elaborate segmentation is needed. One possibility is to merge the scan data is with a reference shell that artificially closes such open porosity. This is possible with simple machined geometries but could be more difficult on more dimensionally variable near-net-shape geometries. 
As can be seen in figure 2 all samples had fractures initiating at surface defects and all initiations were at preexisting defects. It can also be seen that none of the samples had fractures initiated by any of the 10 largest pores segmented in the sample gauge length. This shows that at the relatively high strain/stress level selected for this study, the closeness of the pore to the surface had a higher correlation than the overall size of the pore in determining fracture initiation. This becomes even clearer considering that out of all initiations identified in the fractography only one pore was not at or within a distance of one pore diameter from the surface. A list of sizes expressed in percent of segmented defects with a projected relative diameter smaller than the largest fracture inducing defect is included in table 2. All fractures originated from pores with sizes in the $90^{\text {th }}$ to $100^{\text {th }}$ percentile range. Interestingly, by comparing with figure 3 it is possible to estimate that it is above this size that the plot becomes linear indicating an exponential pore size distribution. This further implies that in modelling fatigue at the stress levels described in this work it is possible to disregard the smallest pores and use extreme value statistical tools for analysis of Gumbel distributions in order to model the remaining data similar to what was done in [4]. This could also allow for lower resolution XCT scans to be used and consequently larger volumes to be scanned without loss of fatigue critical information regarding spherical porosity.

\section{Conclusions}

- Closeness to the surface has been indicated to be a more suggestive factor than size in predicting the location of failure at the stress levels studied.

- Pores of all detectable sizes have seemingly random distribution in the plane perpendicular to the building direction.

- A correlation between EBM building layer thickness and pore size distribution has been shown with thicker building layers melted using a slower scan speed resulting in larger pores.

- Similarly, a difference in pore size distribution between different heights in the build chamber has been indicated with larger pores closer to the base plate of the builds.

- At the tested loads, the life of samples with larger pores was found to be shorter on average than samples with smaller pores.

- All fracture critical defects were found to be in the $90^{\text {th }}$ to $100^{\text {th }}$ percentile range of defects size indicating that it is possible to truncate the data for statistical analysis and modelling without losing critical information.

- As a technique for fatigue critical investigations, XCT can be unreliable as it in the present study was impossible to identify a fatigue critical LOF defect.

\section{Acknowledgement}

This research has been funded by NFFP (the Swedish National Program for Aeronautical Technology). The authors would also like to thank Jonas Kullgren, GKN Aerospace Sweden AB, for his work with the fatigue testing.

\section{References}

[1] Wohlers report 2018, Technical report, Wohler Associates, (2018).

[2] P. Li, D. H. Warner, A. Fatemi, N. Phan, Int. J. Fatigue, 85 (2016) 130-143.

[3] S. Beretta, S. Romano, Int. J. Fatigue, 94 (2017) 178-191.

[4] S. Romano, A. Brandão, J. Gumpinger, M. Gschweitl, S. Beretta, Mater. Design, 131 (2017) 32-48.

[5] R. Cunningham, A. Nicolas, J. Madsen, E. Fodran, E. Anagnostou, M. D. Sangid, A. D. Rollett, Mater. Res. Lett., 5 (2017) 516-525.

[6] A. Yadollahi, N. Shamsaei, Int. J. Fatigue, 98 (2017) 14-31.

[7] M. Seifi, A. Salem, D. Satko, J. Shaffer, J. J. Lewandowski, Int. J. Fatigue, 94 (2017) 263-287.

[8] M. Neikter, F. Forsberg, R. Pederson, M.-L. Antti, P. Åkerfeldt, S. Larsson, P. Jonsén, G. Puyoo, Aeronaut. Aerosp. Open Access J., 2(3) (2018) 139-145.

[9] U. Ackelid, M. Svensson, Proceedings of the Materials Science and Technology Conference, Pittsburgh, PA, USA, (2009), 2529.

[10] N. Hrabe, T. Gnäupel-Herold, T. Quinn, Int. J. Fatigue, 94 (2016) 202-210.

[11] Y. Murakami, M. Endo, Eng. Fract. Mech., 17(1) (1983) 1-15.

[12] Y. Murakami, S. Beretta, Extremes, 2 (1999) 123-147.

[13] K. Chan, M. Koike, R. Mason, T. Okabe, Metall. Mater. Trans. A, 44 (2013) 1010-1022.

[14] D. Greitemeier, C. D. Donne, F. Syassen, J. Eufinger, T. Melz, Mater. Sci. Tech., 32 (2016) 629-634.

[15] S. Palanivel, A. K. Dutt, E. J. Faierson, R. S. Mishra, Mater. Sci. Eng. A, 654 (2016) 39-52.

[16] S. Tammas-Williams, P. J. Withers, I. Todd, P. B. Prangnell, Sci. Rep., 7 (2017), 7308.

[17] G. K. L. Ng, A. E. W. Jarfors, G. Bi, and H. Y. Zheng, Appl. Phys. A, 97(3) (2009) 641-649.

[18] S. Tammas-Williams, H. Zhao, F. Léonard, F. Derguti, I. Todd, P. B. Prangnell, Mater. Charac., 102 (2015) 47-61. 